\title{
The secret's in: Open data is a foreign concept in Canada
}

$\mathrm{T}$ he systemic secrecy in which Health Canada shrouds data is "outdated" and "embarrassing" in comparison with the openness of other countries in delivering government information into the hands of their citizens, say public health advocates and access to information policy wonks.

While the United States and United Kingdom, among other nations, are locked in an "arms race to see who can be more open with their data," Canada remains "totally outside that debate," says open government access expert David Eaves. Like others, he argues that the government seems intent on keeping critical health information and decision-making processes behind closed doors or paywalls which require that people purchase what information is available.

The government's failure to disclose available health data makes it difficult to comprehend the rationale that underpins its decisions, or its priorities, says Bill Jeffery, national coordinator of the Centre for Science in the Public Interest. It's often "very unclear" how Health Canada "decides what's important to work on and what's not important," he says, citing the recent and as-yet unexplained disbanding of the department's Sodium Working Group as an example (www.cmaj.ca/cgi/doi/10.1503/cmaj .109-3811).

"There's evidence to indicate as many as 16000 premature deaths could be prevented by reducing sodium to the levels recommended by the group, but instead they're working on allergy labels, an initiative the department's regulatory impact assessment said would prevent one death per year," Jeffery explains. "The public's entitled to know why they're not taking action, but there's no explanation for their actions, no plan for the future and those 16000 premature deaths a year are still occurring and accumulating."

Data that inform Health Canada's regulatory decision-making processes in

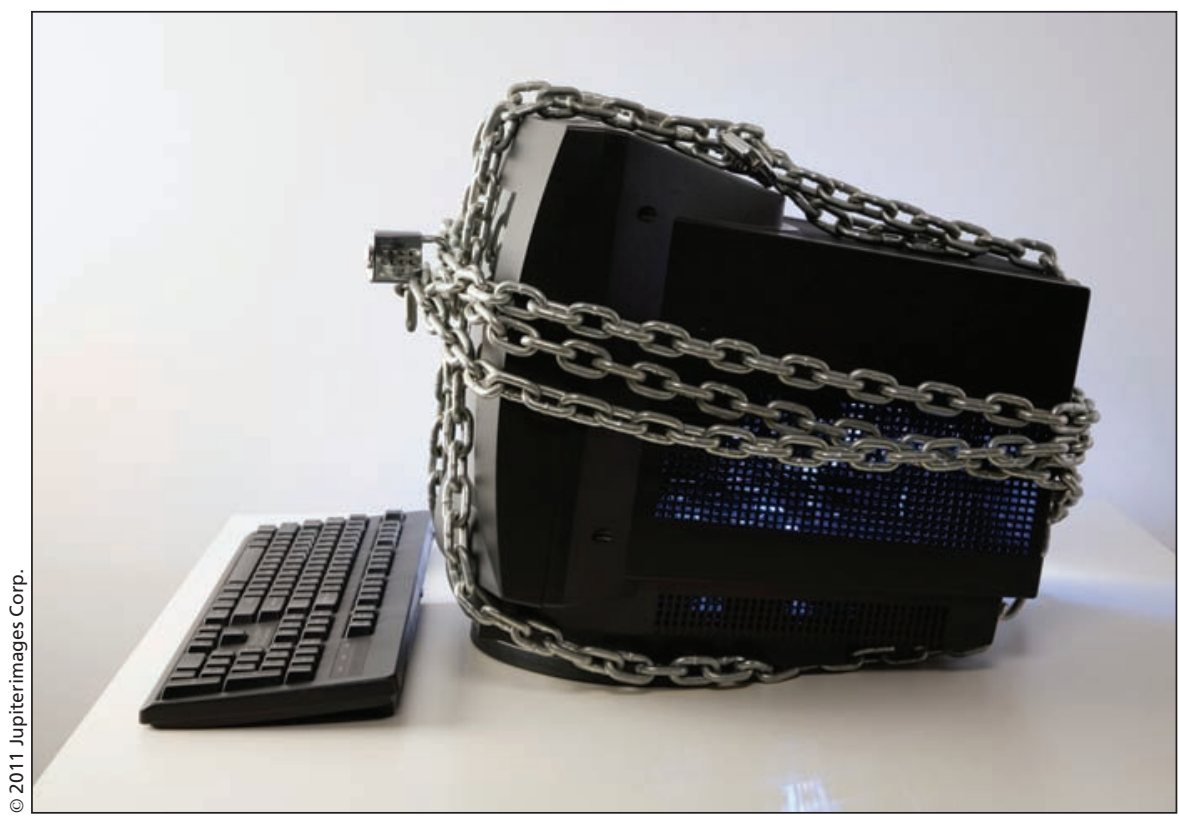

Data that inform Health Canada's regulatory decision-making processes in such areas as drug reviews are also kept under tight control, often in the name of protecting industry interests, experts say.

such areas as drug reviews are also kept under tight control, often in the name of protecting industry interests, says Dr. Joel Lexchin, a professor in the School of Health Policy and Management at York University in Toronto, Ontario.

"Health Canada will not release clinical efficacy and safety data submitted by drug companies regardless of whether or not you submit an access to information request, unless the company approves, the reasoning being such information will provide competitors with an unfair advantage," he explains. "The US is party to all the same trade agreements which say you have to protect confidential business data, so if they can release that kind of [clinical safety and efficacy] information, it's not clear to me why Health Canada cannot do the same."

Even the information that Health Canada is willing to share is often difficult to access, Jeffery says. "With regard to day-to-day disclosures, comparing Canada to the US is like night and day. Documents it would take me two years to get here by access to information request literally take two seconds to obtain from US websites."

Moreover, when information is disclosed, it's often delivered in unsearchable formats, Jeffery adds. "The typical thing the government does is print the data on paper and scan the paper back into the computer, so you end up with a PDF file several thousand pages long that you have to search and extract data from manually, reading the entire document and retyping everything into a spreadsheet yourself. It's an obvious attempt to avoid scrutiny."

Health Canada's notoriously difficult-to-navigate website makes finding even publicly accessible files a "disaster," Lexchin argues. "There are documents I know that are there but the only way I can find them is to put the title in a Google search. Even though I know what I'm looking for, there's no way I could find it if I tried to navigate the site itself."

The case for more open government, particularly in the area of health, is becoming "embarrassingly" clear, says Dr. Carolyn Bennett, the Liberal Party's 
critic for democratic renewal. "I felt jealous in a meeting with the former health minister of Scotland when he told me they had disease outcomes down to postal code, so you could actually deliver needs-based care in individual neighbourhoods. Family doctors in communities could access information about those individuals most at risk or specific health concerns in their areas and target their services accordingly. ... Within three years, they saw measurable improvements in the health outcomes in those neighbourhoods."

But such public health benefits would require a "big culture shift from the government notion that they own the data they collect," says Eaves. "That even comes down to an agency or department level. Forget about sharing information with the public. It's tough enough right now getting information shared within or across governments."

Paywalls on Statistics Canada data are a prime example of that attitude, Bennett says. "Having to purchase data as a city, township, public health agency or department seems wrong when we've already paid for that data with our taxes. For the insignificant amount of money Statistics Canada receives, it builds a very significant barrier to citizens accessing what technically should be public information."

Eaves argues that withholding access to government datasets also stifles potentially life-saving innovations, citing a drug identification tool being developed using pharmaceutical data made accessible under the US Open Government Initiative. "That's literally a life-saving tool and it's the kind of innovation we could have here."

Canada's federal standing committee on access to information, privacy and ethics is now undertaking a study of open government, but Eaves, who testified before the committee in February, is discouraged that Canadian officials seem opposed to the "notion that data should be free."

Bennett concurs. "Apparently Canadian officials when they go to conferences on the issue are only allowed to talk about open data, not open government," she says. "This government doesn't even have a policy, let alone a directive, on open government." Lauren Vogel, CMAJ

CMAJ 2011. DOI:10.1503/cmaj.109-3837 